\title{
Efeito do Nível de Concentrado sobre o Peso dos Órgãos Internos e do Conteúdo Gastrintestinal de Bovinos Nelore Não-Castrados ${ }^{1}$
}

\section{Antonia Sherlânea Chaves Véras ${ }^{2}$, Sebastião de Campos Valadares Filho ${ }^{3}$, José Fernando Coelho da Silva ${ }^{4}$, Mário Fonseca Paulino ${ }^{3}$, Paulo Roberto Cecon ${ }^{5}$, Rilene Ferreira Diniz Valadares ${ }^{6}$, Marcelo de Andrade Ferreira ${ }^{2}$, Cristina Maria Sá Fontes ${ }^{7}$}

RESUMO - O efeito de cinco níveis de concentrado na dieta foi avaliado sobre o conteúdo do trato gastrintestinal e o peso dos órgãos internos e dos compartimentos gastrintestinais de bovinos Nelore não-castrados. Vinte e cinco animais com peso médio de $330 \mathrm{~kg}$ e idade média de 20 meses foram distribuídos, em delineamento inteiramente casualizado, em cinco tratamentos com diferentes níveis de concentrado na matéria seca das dietas $(25,0 ; 37,5 ; 50,0 ; 62,5$; e 75,0\%). Os animais, quando atingiram o peso de abate préestabelecido de $450 \mathrm{~kg}$, foram abatidos e os pesos dos órgãos e das vísceras, registrados. Foram ajustadas equações de regressão para verificar o efeito da inclusão de concentrado na dieta sobre os pesos de cada órgão e, ou, víscera. Os pesos, tanto absolutos, quanto relativos, do conteúdo do trato gastrintestinal diminuíram linearmente, enquanto os pesos do fígado, dos rins e do baço aumentaram linearmente, em função dos níveis de concentrado nas dietas. Os pesos do rúmen-retículo foram influenciados quadraticamente pelos níveis de concentrado, com valores mínimos estimados em $6,45 \mathrm{~kg}$ e 1,60 kg/100 kg de peso vazio, para os níveis de 51,72 e 55,51\% de concentrado nas dietas, em relação aos pesos absoluto e relativo, respectivamente. A quantidade de gordura interna aumentou linearmente com a inclusão de concentrado nas dietas.

Palavras-chave: concentrado, conteúdo gastrintestinal, gordura interna, órgãos internos

\section{Effect of Concentrate Level on the Internal Organs Weight and Gastrintestinal Content of Nellore Bulls}

\begin{abstract}
The effect of five dietary concentrate levels on the gastrintestinal contents and internal organs weight of Nellore bulls was evaluated. Twenty-five animals averaging $330 \mathrm{~kg}$ and 20 months of age were allotted to a completely randomized design, with five treatments constituted by different dietary concentrate levels in dry matter $(25.0,37.5,50.0,62.5$, and $75.0 \%)$. The animals, when reached the pre-established slaughter weight of $450 \mathrm{~kg}$, were slaughtered and the organs and viscera weights were recorded. Regression equations were fitted to determine the effect of dietary concentrate inclusion on each organ weight and, or, viscera. The absolute and relative weights of gastrintestinal tract content linearly decreased, while the liver, kidneys and spleen weights linearly increased, in function of concentrate levels in the diets. The reticulum-rumen weights were quadractily influenced by the concentrate levels, with minimum values estimated in $6.45 \mathrm{~kg}$ and $1,60 \mathrm{~kg} / 100 \mathrm{~kg}$ empty body, for the levels of 51.72 and $55.51 \%$ of concentrate in the diets, in relation to the absolute and relative weights, respectively. The internal fat content linearly increased as dietary concentrate increased.
\end{abstract}

Key Words: concentrate, gastrintestinal content, internal fat, internal organs

\section{Introdução}

Em estudos de exigências energéticas dos animais, a determinação do tamanho relativo de seus órgãos internos é importante, já que diferenças nas partes não-integrantes da carcaça podem induzir variações nos requisitos energéticos para mantença (JONES et al.,1985; BALDWIN et al., 1991; HOOG, 1991; OWENS et al.,1995; e FERRELL e JENKINS, 1998 a,b). O tamanho dos órgãos internos também influencia o rendimento da carcaça (JONES et al., 1985).

FERRELL e JENKINS (1998a,b) relataram que variações na mantença e eficiência do ganho estão, freqüentemente, mais associadas com o peso e a atividade metabólica de órgãos viscerais, como intestino e fígado. Segundo CATON e DHUYVETTER (1997), os tecidos viscerais consomem cerca de 50\% da energia destinada para mantença, enquanto os

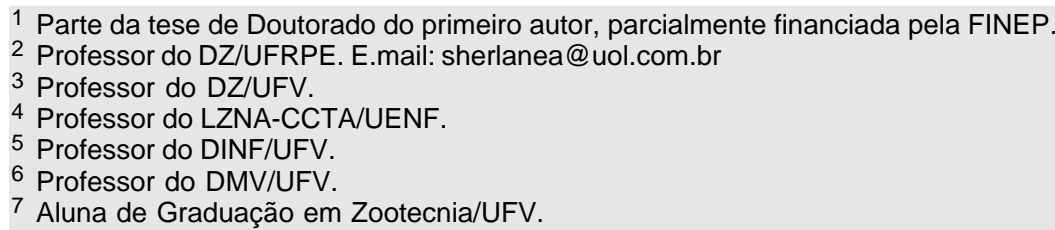


músculos, embora apresentem maior massa no corpo vazio dos animais, consomem apenas $23 \%$ do total da energia para mantença. Isto porque certos tecidos associados com digestão, como o trato gastrintestinal e o fígado, têm maior turnover protéico que o músculo esquelético (OWENS et al., 1993). HOOG (1991) relatou que os tecidos do estômago podem contribuir com mais de $40 \%$ e o fígado, com $18 \%$ da síntese protéica.

Segundo OWENS et al. (1995), o índice mais preciso do conteúdo energético e de nutrientes no corpo animal é o peso do corpo vazio (PCVZ), com os animais submetidos previamente a jejum, porque a influência do conteúdo do trato gastrintestinal (TGI) é eliminada. Alterações no enchimento do trato digestivo e na composição corporal não permitem que o peso total seja bom indicador do crescimento animal (OWENS et al., 1993). ROHR e DAENICKE (1984) relataram que isto é verdadeiro, porque os requisitos para crescimento são altamente relacionados com o desenvolvimento do corpo vazio e a composição química do ganho de peso vazio. O PCVZ consiste na soma dos vários componentes do corpo, exceto o conteúdo gastrintestinal.

Segundo o AGRICULTURAL RESEARCH COUNCIL - ARC (1980), o conteúdo gastrintestinal dos animais apenas pode ser mensurado após seu abate. Sua determinação pode ser efetuada diretamente por pesagem ou, indiretamente, como a diferença entre o peso vivo (PV) e o PCVZ.

ROHR e DAENICKE (1984) relataram que o conteúdo do TGI representa cerca de 5 a $25 \%$ do PV, com valor médio de $15 \%$. Esta variação é atribuída a fatores como raça, peso e estado fisiológico do animal, tipo de dieta e número de horas em jejum a que os animais foram submetidos.

GONÇALVES (1988) e PERON et al. (1993), trabalhando com diferentes grupos genéticos, observaram menores pesos do trato gastrintestinal em animais Nelore que em taurinos e, ou, mestiços, especialmente nos de origem leiteira, sugerindo que os primeiros possuem menor capacidade de ingestão de alimentos em relação aos demais. Da mesma forma, FERRELL e JENKINS (1998b), comparando o peso do TGI de taurinos (Angus e Hereford) e de raças adaptadas aos trópicos (Brahman, Boran e Tuli), submetidos à alimentação restrita e à vontade, verificaram que os animais Brahman apresentaram menor peso do TGI que os taurinos, para os dois níveis de alimentação.

ROBELIN e GEAY (1984) relataram que a con- centração do conteúdo do TGI no PV, logo após o nascimento, representa menos que $50 \mathrm{~g} / \mathrm{kgPV}$, aumentando rapidamente para $200 \mathrm{~g} / \mathrm{kg}$ de $\mathrm{PV}$ em animais pesando entre 200 e $250 \mathrm{~kg}$ e, posteriormente, decresce para $120 \mathrm{~g} / \mathrm{kg}$ em animais com PV de $350 \mathrm{~kg}$. Em termos percentuais, o conteúdo do trato digestivo no início da vida representa menos que $5 \%$ do $\mathrm{PV}$, mas esta porcentagem aumenta rapidamente e alcança, aproximadamente, 20\% do PV em bovinos pesando entre 200 e $250 \mathrm{~kg}$. Entretanto, o enchimento declina até menos de $12 \%$, em animais com PV em jejum de $350 \mathrm{~kg}$.

Segundo BERG e BUTTERFIELD (1976) e OWENS et al. (1995), estas mudanças sugerem que o trato digestivo alcança seu tamanho e peso à maturidade antes dos componentes da carcaça. Contudo, esses valores podem diferir em função das condições a que o PV foi mensurado e, ou, devido à natureza da dieta e à quantidade de alimento consumido.

A equação para relacionar o $\mathrm{PCVZ}$ com o PV, segundo o ARC (1980), é PV=1,09 (PCVZ + a), em que a é calculado a partir do enchimento inicial de um animal com $75 \mathrm{~kg}$ de PCVZ e igual a 4, para dietas que possuem altas proporções de concentrado; 14, para dietas mistas; e 25, para dietas à base de forragens, e corresponde ao enchimento inicial de 150, 300 e $450 \mathrm{~g} / \mathrm{kg}$ PCVZ, respectivamente. Como pode ser observado, os valores de a variam de acordo com o tipo de dieta. Contudo, aquele Conselho enfatizou que o fator 1,09 exclui o caso de animais sujeitos a variações nas dietas, porque considera que aumento no PCVZ é acompanhado por aumento no enchimento. Por outro lado, a equação sugerida pelo NRC (1984): PCVZ = 0,891 PV em jejum, com o enchimento, portanto, igual a 0,109 , não permite observação do aumento do enchimento com o aumento do PCVZ.

O enchimento do TGI é maior quando os animais ingerirem feno que silagem de uma mesma forragem e é proporcional ao tamanho de partículas do volumoso (ROHR e DAENICKE, 1984). Portanto, a adição de concentrado na ração diminui o conteúdo gastrintestinal (ARC, 1980; ROHR e DAENICKE, 1984). Por isto, FERREIRA et al. (2000) concluíram que a melhor maneira de expressar o ganho de peso de bovinos alimentados com diferentes níveis de concentrado na dieta é em relação ao peso vazio, para evitar a influência do conteúdo do TGI, ratificando as colocações de OWENS et al. (1993, 1995).

HOGG (1991) relatou que o estresse nutricional que acarreta manutenção ou perda de peso influencia o peso e a proporção dos órgãos internos, especial- 
1122 Rev. bras. zootec.

mente, fígado, estômago e intestinos. Nesse sentido, PERON et al. (1993) observaram que o peso do TGI foi menor em animais submetidos à restrição alimentar. Da mesma forma, JORGE et al. (1997) relataram menores pesos dos compartimentos do TGI e do fígado de animais estressados nutricionalmente, assim como OLIVEIRA (1999), em animais submetidos a pastejo restrito.

HOGG (1991) afirmou que, em geral, o ganho compensatório é substancialmente atribuído ao aumento do conteúdo do TGI e ao aumento do peso do trato gastrintestinal e de outros órgãos internos.

JONES et al. (1985) compararam os pesos dos órgãos e da gordura visceral em animais mestiços e Holandeses alimentados com dietas à base de concentrado, com dietas à base de forragens, e observaram maiores pesos destes componentes no corpo vazio dos animais cujas dietas tinham maiores proporções de concentrado. Estes autores concluíram que os animais pertencentes à raça Holandesa apresentaram maiores órgãos viscerais, trato digestivo e depósito de gordura visceral, com consequente menor rendimento de carcaça, já que o grau de musculosidade é negativamente correlacionado com o tamanho da cavidade corporal.

Evidências de que a proporção volumoso: concentrado na dieta, o local de deposição de gordura, o tamanho e as taxas metabólicas dos órgãos e tecidos, também influenciam os requisitos energéticos para mantença dos animais foram observadas por vários autores, entre eles, OWENS et al. (1995), que relataram que em raças com aptidão leiteira, os maiores depósitos de gordura encontram-se nos componentes "não-carcaça", ocasionando maior exigência para mantença.

Segundo FERRELL e JENKINS (1998a,b), os órgãos viscerais apresentam elevadas taxas metabólicas e, principalmente o fígado e o trato gastrintestinal são responsivos a alterações na ingestão de alimentos e, juntamente com o aumento no tamanho dos órgãos internos (FOX et al., 1992), respondem em parte pelos maiores requerimentos de animais com potencial para elevada produção de leite.

Diante do exposto, o objetivo deste trabalho foi o de verificar a influência de cinco níveis de concentrado na dieta, sobre o conteúdo do trato gastrintestinal e o peso dos órgãos internos e dos compartimentos gastrintestinais de bovinos Nelore não-castrados.

\section{Material e Métodos}

O local do experimento, as instalações, as dietas (proporção dos ingredientes e composição), o sistema de alimentação e o manejo dos animais foram descritos por VÉRAS et al. (2000).

Foram utilizados 30 animais com peso médio de $330 \mathrm{~kg}$ e idade média de 20 meses. Após período de adaptação de 40 dias, cinco foram abatidos para servirem como referência para estimativa do peso de corpo vazio (PCVZ) inicial dos 25 animais remanescentes, os quais foram distribuídos em cinco tratamentos com diferentes níveis de concentrado nas dietas $(25,0 ; 37,5 ; 50,0 ; 62,5$ e 75,0\%), com base na matéria seca, em delineamento inteiramente casualizado, com cinco repetições.

Pesagens dos animais foram efetuadas a cada 28 dias. Os animais, à medida que se aproximavam do peso pré-estabelecido de abate, de $450 \mathrm{~kg}$, eram pesados em intervalos menores, de forma a serem abatidos próximo ao peso previsto.

Antes do abate, os animais foram submetidos a jejum de 16 horas. Após o abate, o aparelho gastrintestinal de cada animal foi esvaziado e, juntamente com os órgãos, foram lavados e pesados separadamente. Posteriormente, seus pesos foram somados aos das demais partes do corpo (carcaça, cabeça, couro, cauda, pés e sangue), para determinação do PCVZ. A relação obtida entre o PCVZ e o peso vivo (PV) dos animais referência foi utilizada para estimativa do PCVZ inicial dos animais que permaneceram no experimento.

Foram ajustadas equações de regressão para os pesos absolutos e relativos do conteúdo do trato gastrintestinal, dos órgãos internos (coração, pulmões, fígado, rins e baço), das vísceras (rúmenretículo, omaso, abomaso, intestino delgado e intestino grosso) e da gordura interna, em função dos níveis de concentrado nas dietas.

Os resultados foram interpretados estatisticamente por meio de análises de variância e regressão, utilizando-se o Sistema de Análises Estatísticas e Genéticas - SAEG (UNIVERSIDADE FEDERAL DE VIÇOSA - UFV, 1995).

\section{Resultados e Discussão}

Nas Tabelas 1 e 2, são apresentadas as médias, os coeficientes de variação e determinação e as equações ajustadas para os pesos absolutos $(\mathrm{kg})$ e relativos (kg/100 kg do PCVZ) do conteúdo do trato 
Tabela 1 - Médias, coeficientes de variação (CV) e determinação $\left(r^{2}\right)$ e equações de regressão ajustadas (ER) para os pesos absolutos, em kg, do conteúdo do trato gastrintestinal (CTGl), coração, pulmões, fígado, rins e baço de bovinos Nelore, em função dos níveis de concentrado (NC) nas dietas

Table 1 - Means, coefficients of variation (CV) and determination $\left(r^{2}\right)$ and fitted regression equations (RE) of the absolute weights (kg) of the gastrintestinal tract content (GITC), heart, lungs, liver, kidneys and spleen of Nellore bulls, on the concentrate levels (CL) in the diets

\begin{tabular}{|c|c|c|c|c|c|c|c|c|}
\hline \multirow[t]{2}{*}{ Itens } & \multicolumn{5}{|c|}{$\begin{array}{c}\text { Níveis de concentrado ( } \% \text { ) } \\
\text { Levels of concentrate }\end{array}$} & \multirow[t]{2}{*}{$\mathrm{CV}(\%)$} & \multirow[t]{2}{*}{$\mathrm{r}^{2}$} & \multirow[t]{2}{*}{$\begin{array}{l}\mathrm{ER} \\
R E\end{array}$} \\
\hline & 25 & 37,5 & 50 & 62,5 & 75 & & & \\
\hline $\begin{array}{l}\text { CTGI } \\
\text { GITC }\end{array}$ & 73,43 & 63,06 & 60,68 & 52,26 & 50,90 & 12,28 & 0,94 & $\hat{\mathrm{Y}}=82,4104-0,44686^{* *} \mathrm{NC}$ \\
\hline $\begin{array}{l}\text { Coração } \\
\text { Heart }\end{array}$ & 1,44 & 1,46 & 1,51 & 1,54 & 1,42 & 15,08 & - & $\hat{\mathrm{Y}}=1,47$ \\
\hline $\begin{array}{l}\text { Pulmões } \\
\text { Lungs }\end{array}$ & 2,43 & 2,54 & 3,37 & 2,59 & 2,71 & 31,79 & - & $\hat{\mathrm{Y}}=2,72$ \\
\hline $\begin{array}{l}\text { Fígado } \\
\text { Liver }\end{array}$ & 4,69 & 5,22 & 5,55 & 5,79 & 6,59 & 9,30 & 0,96 & $\hat{\mathrm{Y}}=3,8216+0,034892 * * \mathrm{NC}$ \\
\hline $\begin{array}{l}\text { Rins } \\
\text { Kidneys }\end{array}$ & 0,71 & 0,72 & 0,78 & 0,91 & 0,90 & 15,69 & 0,86 & $\hat{\mathrm{Y}}=0,5758+0,004592 * * \mathrm{NC}$ \\
\hline $\begin{array}{l}\text { Baço } \\
\text { Spleen }\end{array}$ & 0,98 & 1,03 & 1,15 & 1,15 & 1,24 & 10,87 & 0,94 & $\hat{\mathrm{Y}}=0,8570+0,005064 * * \mathrm{NC}$ \\
\hline
\end{tabular}

**significativo a $1 \%$ de probabilidade pelo teste $\mathrm{F}$.

${ }^{*}$ significant at $1 \%$ of probability by $F$ test.

gastrintestinal e dos órgãos internos, em função dos níveis de concentrado nas rações, respectivamente. As respostas para as duas formas de expressar as variáveis estudadas foram similares.

O conteúdo do TGI diminuiu linearmente com a inclusão de concentrado nas dietas. Este resultado já era esperado uma vez que o aumento de concentrado na ração reduz o conteúdo gastrintestinal (ARC, 1980;
ROHR e DAENICKE, 1984) e concorda com os relatos de FERREIRA et al. (2000), bem como corrobora com as recomendações dos referidos autores e de OWENS et al. (1993 e 1995), quanto à utilização do PCVZ como índice para expressar o ganho de peso de animais alimentados com vários níveis de concentrado.

Quanto aos pesos do coração e pulmões, estes não foram influenciados pelos níveis de concentrado,

Tabela 2 - Médias, coeficientes de variação (CV) e determinação $\left(r^{2}\right)$ e equações de regressão ajustadas (ER) para os pesos relativos, em kg/100 kg de corpo vazio, do conteúdo do trato gastrintestinal (CTGI), coração, pulmões, fígado, rins e baço de bovinos Nelore, em função dos níveis de concentrado (NC) nas dietas

Table 2 - Means, coefficients of variation (CV) and determination $\left(r^{2}\right)$ and fitted regression equations (RE) of the relative weights $(\mathrm{kg} / 100$ $\mathrm{kg}$ empty body) of the gastrintestinal tract content (GITC), heart, lungs, liver, kidneys and spleen of Nellore bulls, on the concentrate levels $(C L)$ in the diets

\begin{tabular}{|c|c|c|c|c|c|c|c|c|}
\hline \multirow[t]{2}{*}{ Itens } & \multicolumn{5}{|c|}{$\begin{array}{c}\text { Níveis de concentrado ( \% ) } \\
\text { Levels of concentrate }\end{array}$} & \multirow[t]{2}{*}{$\mathrm{CV}(\%)$} & \multirow[t]{2}{*}{$\mathrm{r}^{2}$} & \multirow[t]{2}{*}{$\begin{array}{l}\mathrm{ER} \\
R E\end{array}$} \\
\hline & 25 & 37,5 & 50 & 62,5 & 75 & & & \\
\hline $\begin{array}{l}\text { CTGI } \\
\text { GITC }\end{array}$ & 18,96 & 15,93 & 15,17 & 12,84 & 12,34 & 12,43 & 0,94 & $\hat{\mathrm{Y}}=21,5814-0,13067 * * \mathrm{NC}$ \\
\hline $\begin{array}{l}\text { Coração } \\
\text { Heart }\end{array}$ & 0,37 & 0,37 & 0,38 & 0,38 & 0,34 & 15,19 & - & $\hat{\mathrm{Y}}=0,37$ \\
\hline $\begin{array}{l}\text { Pulmões } \\
\text { Lungs }\end{array}$ & 0,63 & 0,64 & 0,84 & 0,64 & 0,66 & 31,52 & - & $\hat{\mathrm{Y}}=0,68$ \\
\hline $\begin{array}{l}\text { Fígado } \\
\text { Liver }\end{array}$ & 1,21 & 1,32 & 1,39 & 1,42 & 1,60 & 9,22 & 0,95 & $\hat{\mathrm{Y}}=1,0346+0,007036^{* *} \mathrm{NC}$ \\
\hline $\begin{array}{l}\text { Rins } \\
\text { Kidneys }\end{array}$ & 0,18 & 0,18 & 0,20 & 0,22 & 0,22 & 16,84 & 0,83 & $\hat{\mathrm{Y}}=0,15578+0,0009024^{*} \mathrm{NC}$ \\
\hline $\begin{array}{l}\text { Baço } \\
\text { Spleen }\end{array}$ & 0,25 & 0,26 & 0,29 & 0,28 & 0,30 & 12,06 & 0,87 & $\hat{\mathrm{Y}}=0,23041+0,0009315^{* *} \mathrm{NC}$ \\
\hline
\end{tabular}


1124 Rev. bras. zootec.

porque estes órgãos mantêm sua integridade por terem prioridade na utilização de nutrientes, independente do nível de alimentação (PERON et al.,1993; FERREIRA et al., 2000).

Os pesos do fígado, rins e baço aumentaram linearmente em resposta à adição de concentrado na dieta. OWENS et al. (1993) e FERRELL e JENKINS (1998a,b) relataram que principalmente o fígado tem altas taxas metabólicas, porque participa ativamente no metabolismo de nutrientes e, portanto, é responsivo à ingestão de nutrientes. Conforme a massa do fígado aumenta, os requisitos energéticos para mantença também aumentam.

FERREIRA et al. (2000) verificaram aumentos lineares dos pesos do fígado, rins e baço de bovinos $\mathrm{F} 1$ Simental x Nelore, alimentados com níveis crescentes de concentrado. JORGE et al. (1997) e OLIVEIRA (1999) relataram menores pesos do fígado de bovinos submetidos à alimentação restrita. Para os rins, SIGNORETTI et al. (1996) verificaram aumento linear no peso destes órgãos, com a inclusão de concentrado na dieta de bezerros Holandeses.

O efeito dos níveis de concentrado sobre os pesos absolutos e relativos dos compartimentos gastrintestinais e da gordura interna (cavitária e visceral) é apresentado, respectivamente, nas Tabelas 3 e 4.

Os pesos de rúmen-retículo foram influenciados quadraticamente pelos níveis de concentrado, com valores mínimos estimados em 6,45 kg e 1,60 $\mathrm{kg} / 100 \mathrm{~kg}$ de peso vazio, para os níveis de $51,72 \mathrm{e}$ $55,51 \%$ de concentrado nas dietas, em relação aos pesos absoluto e relativo, respectivamente. Pode-se observar que, para o nível de $25 \%$ de concentrado, portanto, para a dieta com maior inclusão de volumoso, os pesos de rúmen-retículo foram maiores.

Os pesos de abomaso, intestino delgado e intestino grosso, nas duas formas em que foram expressos, não foram influenciados pelos níveis de concentrado, bem como o peso absoluto do omaso, cujo valor médio foi $2,36 \mathrm{~kg}$, embora possa ser observado que os valores absolutos diminuíram com a inclusão de concentrado. Quando expresso em relação ao PCVZ (Tabela 4), o peso do omaso diminuiu linearmente com a inclusão de concentrado na dieta. VAN SOEST (1994) relatou que dietas com altos níveis de concentrado provocam involução do omaso.

A gordura interna aumentou linearmente com o aumento de concentrado na ração. Estes resultados são coerentes com o relato de ROBELIN e GEAY (1984) de que o aumento no consumo de energia geralmente leva ao aumento na deposição de gordura no corpo do animal. OWENS et al. (1995) afirmaram que maiores depósitos de gordura cavitária ocasionam maior exigência para mantença.

Tabela 3 - Médias, coeficientes de variação (CV) e determinação $\left(r^{2} / R^{2}\right)$ e equações de regressão ajustadas (ER) para os pesos absolutos, em kg, do rúmen-retículo (RURE), omaso (OMA), abomaso (ABO), intestino delgado (ID), intestino grosso (IG) e gordura interna (GI) de bovinos Nelore, em função dos níveis de concentrado (NC) nas dietas

Table 3 - Means, coefficients of variation (CV) and determination $\left(r^{2} / R^{2}\right)$ and fitted regression equations (RE) of the absolute weights $(\mathrm{kg})$ of the reticulum-rumen (RERU), omasum (OMA), abomasum (ABO), thin intestine (TI), large intestine (LI) and internal fat (IF) of Nellore bulls, on the concentrate levels (CL) in the diets

\begin{tabular}{|c|c|c|c|c|c|c|c|c|}
\hline \multirow[t]{2}{*}{ Itens } & \multicolumn{5}{|c|}{$\begin{array}{c}\text { Níveis de concentrado ( } \% \text { ) } \\
\text { Levels of concentrate }\end{array}$} & \multirow[t]{2}{*}{$\mathrm{CV}(\%)$} & \multirow[t]{2}{*}{$\mathrm{r}^{2} / \mathrm{R}^{2}$} & \multirow[t]{2}{*}{$\begin{array}{l}\mathrm{ER} \\
R E\end{array}$} \\
\hline & 25 & 37,5 & 50 & 62,5 & 75 & & & \\
\hline $\begin{array}{l}\text { RURE } \\
R E R U\end{array}$ & 7,29 & 6,52 & 6,50 & 6,66 & 6,99 & 8,75 & 0,92 & $\hat{\mathrm{Y}}=9,3466-0,111964 * * \mathrm{NC}+0,0010825 * \mathrm{NC}^{2}$ \\
\hline OMA & 2,41 & 2,63 & 2,46 & 2,25 & 2,04 & 18,95 & - & $\hat{\mathrm{Y}}=2,36$ \\
\hline $\begin{array}{l}\mathrm{ABO} \\
\mathrm{ID}\end{array}$ & 1,09 & 1,05 & 1,12 & 1,23 & 1,20 & 13,34 & - & $\hat{\mathrm{Y}}=1,14$ \\
\hline $\begin{array}{l}\text { TI } \\
\text { IG }\end{array}$ & 4,47 & 4,22 & 4,65 & 4,90 & 4,77 & 14,62 & - & $\hat{\mathrm{Y}}=4,60$ \\
\hline $\begin{array}{l}\mathrm{LI} \\
\mathrm{GI}\end{array}$ & 2,85 & 2,67 & 2,89 & 2,82 & 2,55 & 27,00 & - & $\hat{\mathrm{Y}}=2,76$ \\
\hline IF & 3,97 & 4,10 & 4,89 & 5,33 & 5,06 & 19,47 & 0,80 & $\hat{\mathrm{Y}}=3,3086+0,0272 * * \mathrm{NC}$ \\
\hline
\end{tabular}

${ }^{*} \mathrm{e}^{* *}$ significativos a 5 e $1 \%$ de probabilidade, respectivamente, pelo teste $\mathrm{F}$.

${ }^{*}$ and ${ }^{* *}$ significant at 5 and $1 \%$ of probability, respectively, by $F$ test. 
VÉRAS et al.

Tabela 4 - Médias, coeficientes de variação $(C V)$ e determinação $\left(r^{2} / R^{2}\right)$ e equações de regressão ajustadas (ER) para os pesos relativos, em kg/100 kg de corpo vazio, do rúmen-retículo (RURE), omaso (OMA), abomaso (ABO), intestino delgado (ID), intestino grosso (IG) e gordura interna (Gl) de bovinos Nelore, em função dos níveis de concentrado (NC) nas dietas

Table 4 - Means, coefficients of variation $(C V)$ and determination $\left(r^{2} / R^{2}\right)$ and fitted regression equations (RE) of the relative weights $(\mathrm{kg} /$ $100 \mathrm{~kg}$ empty body) of the reticulum-rumen (RERU), omasum (OMA), abomasum (ABO), thin intestine (TI), large intestine (LI) and internal fat (IF) of Nellore bulls, on the concentrate levels (CL) in the diets

\begin{tabular}{|c|c|c|c|c|c|c|c|c|}
\hline \multirow[t]{2}{*}{ Itens } & \multicolumn{5}{|c|}{$\begin{array}{c}\text { Níveis de concentrado ( } \% \text { ) } \\
\text { Levels of concentrate }\end{array}$} & \multirow[t]{2}{*}{$\mathrm{CV}(\%)$} & \multirow[t]{2}{*}{$\mathrm{r}^{2} / \mathrm{R}^{2}$} & \multirow[t]{2}{*}{$\begin{array}{l}\mathrm{ER} \\
R E\end{array}$} \\
\hline & 25 & 37,5 & 50 & 62,5 & 75 & & & \\
\hline $\begin{array}{l}\text { RURE } \\
R E R U\end{array}$ & 1,88 & 1,65 & 1,63 & 1,64 & 1,69 & 8,89 & 0,92 & $\hat{\mathrm{Y}}=2,4654-0,0311438 * * \mathrm{NC}+0,0002805 * \mathrm{NC} 2$ \\
\hline OMA & 0,62 & 0,67 & 0,61 & 0,55 & 0,49 & 19,11 & 0,77 & $\hat{\mathrm{Y}}=0,737985-0,00296848 * \mathrm{NC}$ \\
\hline $\mathrm{ABO}$ & 0,28 & 0,26 & 0,28 & 0,30 & 0,29 & 12,90 & - & $\hat{\mathrm{Y}}=0,28$ \\
\hline $\begin{array}{l}\mathrm{ID} \\
T I\end{array}$ & 1,15 & 1,07 & 1,16 & 1,20 & 1,15 & 14,00 & - & $\hat{\mathrm{Y}}=1,15$ \\
\hline $\begin{array}{l}\text { IG } \\
L I\end{array}$ & 0,73 & 0,67 & 0,72 & 0,69 & 0,61 & 26,58 & - & $\hat{\mathrm{Y}}=0,69$ \\
\hline $\begin{array}{l}\mathrm{GI} \\
I F\end{array}$ & 1,02 & 1,03 & 1,21 & 1,30 & 1,23 & 18,77 & 0,72 & $\hat{\mathrm{Y}}=0,8901+0,00543416 * \mathrm{NC}$ \\
\hline
\end{tabular}

\section{Conclusões}

Os níveis de concentrado na dieta influenciaram os pesos do fígado, dos rins e do baço.

Os pesos de rúmen-retículo foram influenciados quadraticamente pelos níveis de concentrado, com pesos mínimos estimados de $6,45 \mathrm{~kg}$ e $1,60 \mathrm{~kg} / 100 \mathrm{~kg}$ de corpo vazio para os níveis de 51,72 e $55,51 \%$ de concentrado, respectivamente, para os pesos relativo e absoluto.

A quantidade de gordura interna aumentou linearmente com a inclusão de concentrado.

\section{Referências Bibliográficas}

AGRICULTURAL RESEARCH COUNCIL - ARC. 1980. The nutrient requirements of ruminants livestock. London: Commonwealth Agricultural Bureaux. 351p.

BALDWIN, R.L., CALVERT, C.C., OBERBAUER, A.M. 1991. Growth control in future. In: PEARSON, A.M. DUTSON, T.R. (Eds). Growth regulation in farm animals. London: Elsevier Applied Science. p.589-613.

BERG. R.T., BUTTERFIELD, R.M. 1976. New concepts of cattle growth. New York: Sydney University. 240p.

CATTON, J.S., DHUYVETTER, D.V. 1997. Influence of energy supplementation on grazing ruminants: requirements and responses. J. Anim. Sci., 75:533-542.

FERREIRA, M.A., VALADARES FILHO, S.C., MUNIZ, E.B. et al. 2000. Características das carcaças, biometria do trato gastrintestinal, tamanho dos órgãos internos e conteúdo gastrintestinal de bovinos F1 Simental x Nelore alimentados com vários níveis de concentrado. Rev. bras. zootec. 29(4):1174-1182.
FERRELL, C.L., JENKINS, T.G. 1998a. Body composition and energy utilization by steers of diverse genotypes fed a high-concentrate diet during the finishing period: I. Angus, Belgian Blue, Hereford, and Piedmontese Sires. J. Anim. Sci., 76:637-646.

FERRELL, C.L., JENKINS, T.G. 1998b. Body composition and energy utilization by steers of diverse genotypes fed a highconcentrate diet during the finishing period: I. Angus, Belgian Blue, Hereford, and Piedmontese Sires. J. Anim. Sci., 76:647-657.

FOX, D.G., SNIFFEN, C.J., O'CONNOR, J.D. et al. 1992. A net carbohydrate and protein system for evaluating cattle diets: III. Cattle requirements and diet adequacy. J. Anim. Sci., 70(11):3578-3596.

GONÇALVES, L.C. Digestibilidade, composição corporal, exigências nutricionais e características das carcaças de zebuínos, taurinos e bubalinos. Viçosa, MG: UFV, 1988. 238p. Tese (Doutorado em Zootecnia) - Universidade Federal de Viçosa, 1988.

HOOG, B.W. 1991. Compensatory growth in ruminants. In: PEARSON, A. M. DUTSON, T. R. (Ed). Growth regulation in farm animals. London: Elsevier Applied Science. p.103-134.

JONES, S.D.M., ROMPALA, R.E., JEREMIAH, L.E. 1985. Growth and composition of the empty body in steers of different maturity types fed concentrate or forage diets. J. Anim. Sci., 60(2):427-433.

JORGE, A.M., FONTES, C.A.A., PAULINO, M.F. Efeito da raça e do nível nutricional sobre o tamanho de órgãos internos em zebuínos. In: REUNIÃO ANUAL DA SOCIEDADE BRASILEIRA DE ZOOTECNIA, 34, Juiz de Fora, MG, 1997. Anais ... Juiz de Fora: SBZ, 1997a, p. 466-468.

NATIONAL RESEARCH COUNCIL - NRC. 1984. Nutrient requeriments of beef cattle. 6.ed. Washington, D.C. 90p.

OLIVEIRA, R.C. Ganho de peso, características de carcaça e composição corporal de novilhos, em regime de pastejo, em capim-elefante, durante a estação chuvosa. Viçosa, MG: 
1126 Rev. bras. zootec.

UFV. 1999. Dissertação (Mestrado em Zootecnia) - Universidade Federal de Viçosa, 1999.

OWENS, F.N., DUBESKI, P., HANSON, C.F. 1993. Factors that alter the growth and development of ruminants. J. Anim. Sci., 71:3138-3150.

OWENS, F.N., GILL, D.R., SECRIST, D.S. et al. 1995. Review of some aspects of growth and devepopment of feedlot cattle. J. Anim. Sci., 73:3152-3172.

PERON, A.J., FONTES, C.A.A., LANA, R.P. et al. 1993.Tamanho dos órgãos internos e distribuição da gordura corporal em novilhos de cinco grupos genéticos, submetidos à alimentação restritae äd libitum”. R. Soc. Bras. Zootec., 22(5):813-819.

ROBELIN, J., GEAY, Y. 1984. Body composition of cattle as affected by physiological status, breed, sex and diet. In: GILCHRIST, F.M.C., MACKIE, R.I (Eds.). Herbage nutrition in the subtropics and tropics. Johannesburg: Science Press. p.525-547.

ROHR, K.R., DAENICKE, R. 1984. Nutritional effects on the distribuition of live weight as gastrointestinal tract fill and tissue components in growing cattle. J. Anim. Sci., 38(3):753-765.
SIGNORETTI, R.D., ARAÚJO, G.G.L., COELHO DA SILVA, J.F. et al. Biometria do trato gastrintestinal e tamanho dos órgãos internos de bezerros Holandeses alimentados com quatro níveis de concentrado. In: REUNIÃO ANUAL DA SOCIEDADE BRASILEIRA DE ZOOTECNIA, 33, 1996. Fortaleza. Anais... Fortaleza: SBZ, 1996. p. 402-404.

VAN SOEST, P.J. 1994. Nutritional ecology of the ruminant. Ithaca: Cornell University Press. 2 ed. 476p.

VÉRAS, A.S.C., VALADARES FILHO, S.C., COELHO DA SILVA, J.F. et al. 2000. Consumo e digestibilidade aparente em bovinos Nelore, não-castrados, alimentados com rações contendo diferentes níveis de concentrado. Rev. bras. zootec., 29(6):2367-2378 (supl. 2).

Recebido em: 14/12/99

Aceito em: 21/02/01 